\title{
Minimal statistical-mechanical model for multihyperuniform patterns in avian retina
}

\author{
Enrique Lomba $\odot,{ }^{1}$ Jean-Jacques Weis, ${ }^{2}$ Leandro Guisández, ${ }^{1,3}$ and Salvatore Torquato ${ }^{4,5}$ \\ ${ }^{1}$ Instituto de Química Física Rocasolano, CSIC, Calle Serrano 119, E-28006 Madrid, Spain \\ ${ }^{2}$ Université de Paris-Saclay, Laboratoire de Physique Théorique, Bâtiment 210, 91405 Orsay Cedex, France \\ ${ }^{3}$ IFLYSIB (UNLP, CONICET), 59 No. 789, B1900BTE La Plata, Argentina \\ ${ }^{4}$ Department of Chemistry, Princeton University, Princeton, New Jersey 08544, USA \\ ${ }^{5}$ Princeton Institute for the Science and Technology of Materials, Princeton University, Princeton, New Jersey 08544, USA
}

(Received 4 May 2020; accepted 22 June 2020; published 15 July 2020)

\begin{abstract}
Birds are known for their extremely acute sense of vision. The very peculiar structural distribution of five different types of cones in the retina underlies this exquisite ability to sample light. It was recently found that each cone population as well as their total population display a disordered pattern in which long-wavelength density fluctuations vanish [Jiao et al., Phys. Rev. E 89, 022721 (2014)]. This property, known as hyperuniformity, is also present in perfect crystals. In situations like the avian retina in which both the global structure and that of each component display hyperuniformity, the system is said to be multihyperuniform. In this work, we aim at devising a minimal statistical-mechanical model that can reproduce the main features of the spatial distribution of photoreceptors in avian retina, namely the presence of disorder, multihyperuniformity, and local heterocoordination. This last feature is key to avoiding local clustering of the same type of photoreceptors, an undesirable feature for the efficient sampling of light. For this purpose, we formulate a minimal statisticalmechanical model that definitively exhibits the required structural properties: an equimolar three-component mixture (one component to sample each primary color: red, green, and blue) of nonadditive hard disks to which a long-range logarithmic repulsion is added between like particles. Interestingly, a Voronoi analysis of our idealized system of photoreceptors shows that the space-filling Voronoi polygons display a rather uniform area distribution, symmetrically centered around that of a regular lattice, a structural property also found in human retina. Disordered multihyperuniformity offers an alternative to generate photoreceptor patterns with minimal long-range concentration and density fluctuations. This is the key to overcoming the difficulties in devising an efficient visual system in which crystal-like order is absent.
\end{abstract}

DOI: 10.1103/PhysRevE.102.012134

\section{INTRODUCTION}

Sampling light is one of the essential activities that enables living organisms to interact with the surrounding environment. From simple devices such as the stigma that provides "vision" in certain classes of microalgae [1], to the sophisticated compound eyes of arthropods [2,3], living organisms have developed increasingly efficient ways to map visual information from the external world onto signals that can be processed by their cognitive systems. The case of arthropod eyes is particularly interesting. It is known from classical sampling theory [4] that an optimal sampling of light can be achieved by a hexagonal array of photodetectors. This is actually the pattern adopted by ommatidia (the optical units forming a compound eye) in arthropods. Compound eyes are imaging systems with low aberration, a wide-angle field of view, and infinite field depth [5]. These properties have motivated intense research into the development of bionic compound eyes intended for small robots [6] or sensors for digital cameras [5].

When it comes to vertebrates, with the exception of some teleost fish [7,8] and some reptiles [9], the situation is different, and structural disorder in photoreceptor patterns is the general trend. From this standpoint, birds are in a class of their own. They possess one of the most elaborate visual systems among vertebrates. In avian retina, one can find five different types of cones [10-14]. In addition, 10-20\% of the rods are responsible for night and peripheral vision-being mostly absent from the central area of the retina [15]. In contrast with the regular shape of ommatidia in insects, photoreceptors in bird retina are polydisperse in both size and number $[16,17]$. This variation provides an adaptive advantage; by changing the relative numbers and even the pigmentation of the cones, bird species can have visual capabilities that are adapted to different habitats (sea birds have a high density of red/yellow cones for hazy conditions, nocturnal birds have an extremely high density of luminance double cones, etc.). However, polydispersity is known to frustrate crystallization [18], so an alternative to the regular hexagonal pattern of arthropod eyes is needed if we want to preserve a good sampling of light. In this regard, Jiao and co-workers [8] found that the spatial distribution of photoreceptors in chicken retina retained some "hidden order" reminiscent of crystalline patterns. Namely, they found that long-range density and concentration fluctuations were vanishingly small, i.e., the patterns are hyperuniform [19].

Hyperuniformity can be quantified by means of two intimately connected structural properties. In two dimensions, the number variance of cones associated with a spherical sampling window of radius $R$, defined as $\sigma_{N}^{2}(R)=\left\langle N^{2}\right\rangle_{R}-\langle N\rangle_{R}^{2}$, 
where $N$ is the number of cones contained in the window of radius $R$, and $\langle\cdots\rangle_{R}$ denotes an average over sampling windows. A 2D system is hyperuniform if $\sigma_{N}^{2}(R)$ grows more slowly than $R^{2}$, which is the scaling for ordinary disordered patterns. In Ref. [8], it was found that this quantity obeys the following hyperuniform large- $R$ asymptotic scaling:

$$
\sigma_{N}^{2}(R) \propto R
$$

in the plane. This is one of the possible scalings of hyperuniform systems, also characteristic of crystalline-like order in two dimensions (class I following Ref. [20]). Secondly, it is known that density fluctuations in Fourier space are directly related to the structure factor. This is defined for a set of points/particles with number density $\rho$ by

$$
S(\mathbf{Q})=1+\rho \tilde{h}(\mathbf{Q})
$$

where $\mathbf{Q}$ is the wave vector, and $\tilde{h}(\mathbf{Q})$ is the spatial Fourier transform of $h(r)=g_{2}(r)-1$, with $g_{2}(r)$ the pair distribution function of the point/particle configuration. Equivalently, a hyperuniform system is one in which $S(\mathbf{Q})$ tends to zero as the wave number $Q \equiv|\mathbf{Q}|$ tends to zero. It is possible to show [20] that for a statistically homogenous and isotropic hyperuniform system satisfying Eq. (1) in two dimensions,

$$
S(Q) \propto Q^{\alpha} \quad(Q \rightarrow 0)
$$

with $\alpha>0$. Since Eq. (1) holds for each cone distribution, we will have a relation like (3) for the structure factor computed from each cone pattern, as was found by Jiao and co-workers [8], i.e.,

$$
\lim _{Q \rightarrow 0} S_{i i}(Q)=0
$$

for each cone type $i$. This implies that density fluctuations of the corresponding point patterns will vanish for long wavelengths, i.e., when $Q \rightarrow 0$. The same applies to the overall point pattern. This property was termed "multihyperuniformity" in Ref. [8].

Interestingly, since Torquato and Stillinger [19] introduced the concept of hyperuniformity and stressed its significance in structurally disordered materials, such exotic "states of matter" have been found in a wide variety of systems. A partial list of examples includes amorphous dielectric networks with large and complete photonic band gaps [21,22], dense transparent disordered media [23], the enhanced pinning of vortices in arrays in superconductors [24], certain composites with desirable transport, dielectric, and fracture properties [25-28], sand piles and other avalanche models $[29,30]$, driven nonequilibrium granular and colloidal systems [31-33], and even immune system receptors [34]; all of these examples have in common the presence of hyperuniformity.

One might ask why hyperuniformity plays such a crucial role in the quality of vision in birds. As mentioned, the optimal sampling configuration of photoreceptors corresponds to a perfectly regular hexagonal arrangement. Hyperuniformity prevents long-wavelength fluctuations in the photoreceptor density (or a concentration of different species) that would otherwise be present in a structurally disordered configuration of photoreceptors. The presence of such fluctuations is certainly not a desirable property for an accurate image representation. Perfectly regular arrangements such as the hexagonal patterns of ommatidia are hyperuniform, but in the case of bird retina, crystal-like order is preempted by polydispersity. Thus disordered hyperuniform patterns might well be a good compromise solution. Multihyperuniformity will guarantee the same sampling quality for each type of photoreceptors and aids in ensuring local heterocoordination, which is key to preventing the unwanted clustering of same color photoreceptors.

After these considerations, it is our aim to build a minimal statistical mechanical model that can reproduce the main characteristics of the photoreceptor distribution. In addition to disorder, these characteristics include multihyperuniformity on the one hand, and local heterocoordination on the other. By this we mean that photoreceptors of the same type should not be allowed to cluster together if color sensitivity is to be uniformly distributed on space. In fact, in Ref. [35] it was shown that a system can be multihyperuniform and display a strong degree of clustering (chain formation in Ref. [35]). From images of actual chicken cone distributions (see Fig. 1 in Ref. [13]) it is readily apparent that cones of different types tend to cluster together, i.e., their spatial distribution displays local heterocoordination. In contrast with heterocoordination, multihyperuniformity is not a local property; it implies the presence of long-range density/concentration correlations for each component.

The findings of Ref. [35] suggest that a mixture with logarithmic long-range repulsions and nonadditive hard-core volume exclusions can display the desired characteristics. Nonadditivity in this context means that (i) the exclusion diameters between unlike particles differ from the arithmetic mean of those between like particles, and (ii) long-range repulsions between unlike particles are different from the geometric mean of the potential between like particles. This implies that cross interactions do not fulfill the LorentzBerthelot (LB) mixing rules. To properly account for the presence of heterocoordination, both the long-range and the short-range hard-core repulsions have to be nonadditive. In the latter instance, the imbalance between unlike and like particle repulsions leads to heterocoordination at low densities. When packing effects become dominant, it is the steric effect of negative nonadditivity that leads to local heterocoordination. Strictly speaking, the model in Refs. [35,36] is a two-dimensional Coulomb plasma. Obviously, in our case the logarithmic repulsion is to be thought of as an effective interaction between photoreceptor cells to mimic "real" conecone interactions. These are mediated by complex cellular entities, and their genesis is unknown to biologists.

It is instructive to compare our model with the multiscale packing model proposed by Jiao et al. [8]. As in the present instance, the multiscale model has two interaction ranges, namely a hard-core repulsion (polydisperse and additive) and a soft-core long-range repulsion. Polydispersity prevents crystallization (as negative nonadditivity does in our model), and the soft-core repulsion leads to multihyperuniformity. To that aim, the parameters of the soft-core interaction are tuned by means of an inverse Monte Carlo optimization algorithm that forces the model's structure factor to agree with the experimental data. In our model, multihyperuniformity is an immediate consequence of the long range and nonadditivity of the logarithmic interactions. Note that, additionally, the 
long-range multiscale interactions vanish for separations beyond $\sqrt{2 /\left(3^{1 / 2} \rho\right)}$, and this means the results will not be strictly hyperuniform. This is due to the fact that in a disordered mixture, the interaction potential must comply with a certain long-range asymptotic behavior [35] [see Eq. (7) below], which is not the case in the multiscale model. Interestingly, both models yield a small wave-number decay for the structure factor of the entire cone population $\sim Q^{2}$. The experimental values seem to agree better with a linear decay [8], although the statistical uncertainties are too large to make a definite conclusion. Interestingly, three-dimensional Coulomb plasmas confined in a plane are known to have structure factors that decay linearly with the wave number as $Q \rightarrow 0$ [37], by which our model could easily be transformed into one that reproduces the apparent experimental decay.

Finally, we will see that a Voronoi analysis of the disordered hyperuniform patterns further illustrates the hidden connection between these and the fully ordered crystal structures. The area distribution of Voronoi polygons is relatively uniform and centered around that of a crystal-like pattern. This uniformity, also found in the Voronoi tessellation of photoreceptors in human retina [38], is in our case the result of the presence of a long-ranged monotonic repulsive interaction.

\section{MODEL AND METHODS}

As mentioned, our minimal model of "retina" consists of three classes of photoreceptors [red-green-blue (RGB)] in which, following Ref. [35], interactions will be defined in terms of a purely repulsive logarithmic potential. In addition, in order to guarantee heterocoordination from moderate to high densities, the particles will have a hard-core volume exclusion defined by a hard-disk diameter $\sigma$, with unlike particles having a distance of minimum approach $(1+\Delta) \sigma$, with $\Delta<0$. From Ref. [35], we know that $\Delta>0$ induces the formation of stable clusters of like particles due to the combination of long-range-like particle repulsions and an effective short-range attraction between like particles due to volume effects. It is worth stressing that in our "minimal model," for computational simplicity, the number of components is reduced to the minimum, three. One can straightforwardly extend the model to four [cyan-magenta-yellow-black (CMYK)] or five types (including the luminance cones as in bird retina) of cones. Leaving aside the rods, whose distribution is highly inhomogeneous (being almost absent from the central area of the retina), the polydispersity introduced by the different cone types is not too large. Therefore, no significant qualitative difference in the results is to be expected by reducing the number of cone types to the minimum, i.e., RGB.

The net interaction between particles of type $i$ and $j$ can be explicitly written as

$$
\beta u_{i j}(r)= \begin{cases}\infty & \text { if } r<\left[1+\Delta\left(1-\delta_{i j}\right)\right] \sigma, \\ -\gamma_{i j} \ln r / \sigma & \text { if } r \geqslant\left[1+\Delta\left(1-\delta_{i j}\right)\right] \sigma,\end{cases}
$$

where $\gamma_{i j}$ is an effective coupling parameter, and $\delta_{i j}$ is Kronecker's symbol. Our minimal model is fully symmetric, with $u_{i i}=u_{11} \forall i$ and $u_{i j}=u_{12} \forall i \neq j$. For the logarithmic repulsion, the coupling parameter is expressed as

$$
\gamma_{i j}= \begin{cases}\Gamma & \text { if } i=j, \\ \lambda \Gamma & \text { if } i \neq j,\end{cases}
$$

with $0 \leqslant \lambda \leqslant 1$. The parameter $\lambda$ controls the nonadditivity of the long-range interactions. Setting $\lambda<1$ leads to a multihyperuniform system, whereas $\lambda=1$ produces a globally hyperuniform system in which individual photoreceptor patterns are not hyperuniform (see Fig. 2 below).

Now, from our study on binary mixtures in Refs. [35,36] we know that disordered systems with long-ranged repulsive interactions, whose small wave-number scaling in Fourier space follows

$$
\lim _{Q \rightarrow} \beta \tilde{u}_{i j}(Q) \propto Q^{-\alpha}
$$

with $\alpha>0$, will exhibit hyperuniformity. In Ref. [35] we found the conditions that cross interactions must fulfill for a binary system to be multihyperuniform. Here we extend our analysis, based on the Ornstein-Zernike (OZ) theory for mixtures, to multicomponent systems. A detailed presentation can be found in Appendix A. Our key result here is that an $n$-component system, in which the small wave-number behavior of the particle-particle interactions follows (7), will be multihyperuniform-i.e., comply with Eq. (4)—if

$$
\lim _{Q \rightarrow 0}|\tilde{\mathbf{u}}(Q)| \neq 0
$$

where $|\cdots|$ denotes a matrix determinant, and the elements of the matrix $\tilde{\mathbf{u}}(Q)$ are the Fourier transform of the speciesspecies interactions, $u_{i j}(r)$. It can be shown that a sufficient condition for Eq. (8) to be fulfilled is that

$$
\lim _{Q \rightarrow 0}\left[\tilde{u}_{i i}(Q) \tilde{u}_{j j}(Q)-\tilde{u}_{i j}(Q)^{2}\right] \neq 0,
$$

which actually means that cross interactions must not comply with the Lorentz-Berthelot mixing rules in the longwavelength limit. We had already found this for binary mixtures in Ref. [35]. In practice, for our model system this means that $\lambda<1$. Here we will simply set $\lambda=0$, which reduces cross interactions to bare hard-core repulsions. The degree of unlike particle aggregation can be tuned at will by modifying the value of the $\lambda$-parameter: the larger its value, the larger the unlike particle repulsion. As $\lambda \rightarrow 1$, the local heterocoordination disappears.

The low- $Q$ asymptotics of the structure factor when all densities are identical $\left(\rho_{i}=\rho / 3 \forall i\right)$ simplifies considerably. A detailed derivation based on the low- $Q$ expansion of the $\mathrm{OZ}$ equation can be found in Appendix B. In our particular case, given the symmetry of the interactions and compositions and setting $\lambda=0$, from Eq. (B2) in Appendix B the limiting behavior of the partial structure factors reduces to

$$
\begin{aligned}
& \lim _{Q \rightarrow 0} S_{i i}(Q)=Q^{2} /(2 \pi \rho \Gamma), \quad \forall i, \\
& \lim _{Q \rightarrow 0} S_{i j}(Q)=\rho \tilde{c}_{i j}^{R}(0) Q^{4} /(2 \pi \rho \Gamma)^{2}, \forall i \neq j,
\end{aligned}
$$

with $\tilde{c}_{i j}^{R}(Q)$ being the Fourier transform of the short-range component of the direct correlation function (see Appendix B for further details), which is nonzero and finite as $Q \rightarrow 0$. When considering mixtures, it is important to monitor the global hyperuniformity using the the number-number structure factor. This is simply the net structure factor given by Eq. (2) where the pair distribution function is computed using all particle types. In practice, it can also be computed from the 


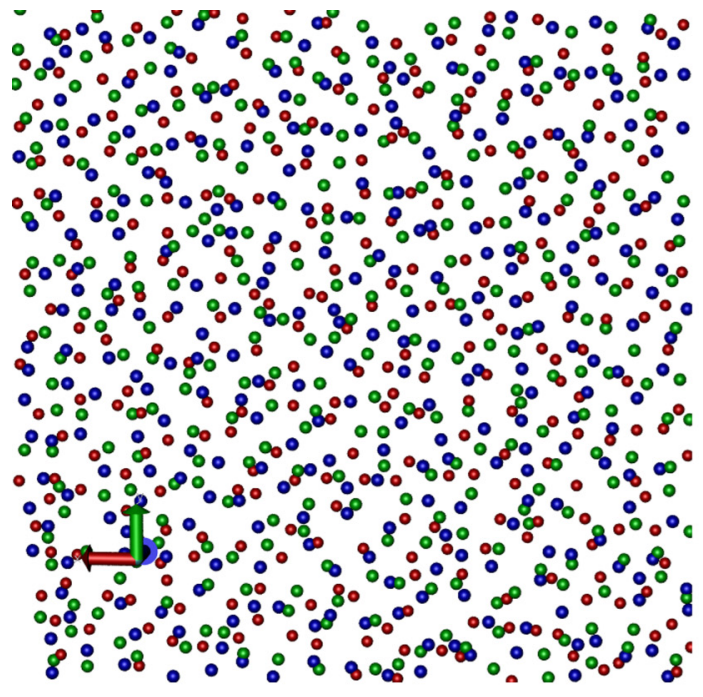

(a)

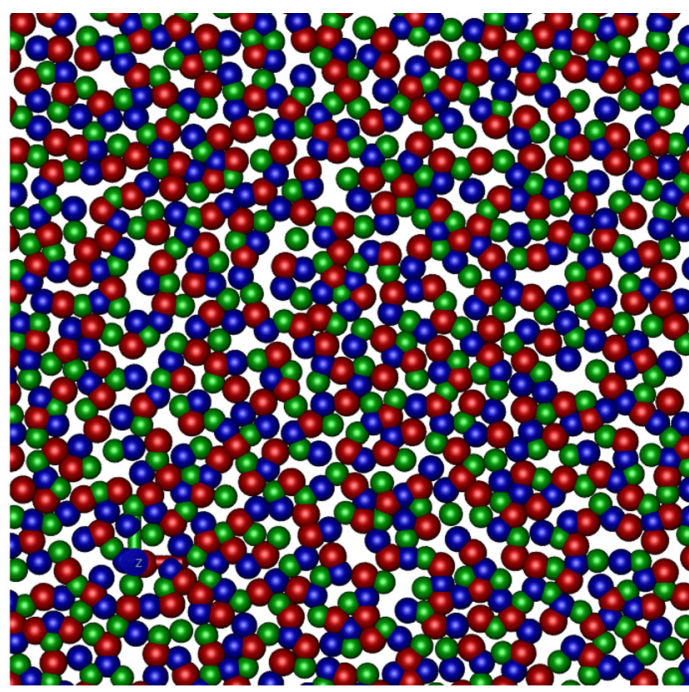

(b)

FIG. 1. Snapshots of Monte Carlo configurations of our three-component minimal model of retina with RGB receptors with $\rho \sigma^{2}=0.2$ (left) and $\rho \sigma^{2}=0.8$ (right). The interaction is defined by a coupling $\gamma=5$ and a nonadditivity parameter $\Delta=-0.2$.

addition of the partial structure factors as

$$
S_{\mathrm{NN}}(Q)=\sum_{i, j} S_{i j}(Q)
$$

From Eq. (10), we then have

$$
\lim _{Q \rightarrow 0} S_{\mathrm{NN}}(Q)=3 Q^{2} /(2 \pi \rho \Gamma)+b Q^{4}
$$

where $b=3 \rho \tilde{c}_{i j}^{R}(0) /(2 \pi \rho \Gamma)^{2}$.

The systems studied in this work have been analyzed using an integral equation approach based on the OZ equation, Eq. (A1) in Appendix A, with a reference hypernetted chain (RHNC) closure [Eq. (11) in Ref. [36]]. We refer the reader to [36] for further details on the numerical approach to solve this equation. We have also performed extensive canonical (NvT) Monte Carlo simulations, in which the energy of the periodic system is evaluated using the Ewald technique with conducting boundary conditions [36,39]. Computational details of the simulations are identical to those of Ref. [36].

\section{RESULTS}

We will first consider two instances of photoreceptor patterns for low density $\left(\rho \sigma^{2}=0.2\right)$ and moderate density $\left(\rho \sigma^{2}=0.8\right)$, with an interspecies hard-core exclusion defined by $\Delta=-0.2$ (i.e., $\sigma_{i j}=0.8 \sigma_{i i}$ ). The coupling factor of the long-range interaction is set to $\Gamma=5$, and the long-range cross interactions are set to zero [i.e., $\lambda=0$ in (6)]. This means that unlike particles will only interact via a pure hardcore exclusion. For comparison, we will also show results for $\lambda=1$, which will only display global hyperuniformity. Partial densities for each photoreceptor type are $\rho \sigma^{2} / 3$.

Two characteristic Monte Carlo snapshots of the lowand high-density multihyperuniform systems are presented in Fig. 1. One can appreciate in the snapshot of Fig. 1(a) for $\rho \sigma^{2}=0.2$ that photoreceptors of different type tend to aggregate in clusters with heterocoordination. These clusters form a low-density fluidlike structure, with average inter- cluster distances $\approx 3-4 \sigma$. When comparing this illustration with real representations of bird cone distributions (see Fig. 1 in Ref. [13]), the similarity is evident. At higher densities $\left(\rho \sigma^{2}=0.8\right)$, packing effects become dominant and clustering is not so apparent, but heterocoordination is still clearly seen in the snapshot of Fig. 1(b). The cluster-size distribution (not shown) is monotonously decreasing, with no dominant cluster size. This is a consequence of the lack of a competing short-range attraction that would counteract the long-range repulsion and would thus stabilize finite-size clusters, as was the case for $\Delta>0$ in Ref. [35].

\section{A. Structure factor analysis}

In Fig. 2, we plot the partial and total structure factors corresponding to the systems described above. The multihyperuniform character of the system is clearly illustrated by their vanishing behavior for low- $Q$. In the insets, one can observe that they closely follow the asymptotic behavior described by Eqs. (10) and (12). Theory and simulation agree to a very large extent.

For comparison, we also plot the theoretical results for $\rho \sigma^{2}=0.2$ and $\lambda=1$. Now, this choice of the long-range cross interactions leads to a globally hyperuniform configuration, as confirmed by the behavior of $S_{\mathrm{NN}}(Q)$ as $Q \rightarrow 0$. In contrast, the partial structure factor does not vanish for $Q \rightarrow 0$ (which rules out multihyperuniformity). Given the low density, the result is close to that of an ideal gas, for which $S_{i i}(Q) \approx x_{i} \forall Q$. Reducing $\lambda$, which actually implies decreasing (or in our present case, eliminating) the unlike long-range repulsive interactions, induces a certain degree of clustering between unlike particles. This effect is visible when comparing the total structure factor at low density (lower graph, red curve in Fig. 2) for $\lambda=0$ and 1. Only in the case of $\lambda=0$ does $S_{\alpha \alpha}(Q)$ exhibit a prepeak at $Q \sigma \approx 1.9$. This reflects the presence of clustering with a correlation length of $\approx 3.2 \sigma$, which we have already qualitatively detected in the snapshot of Fig. 1(a). In summary, the combination of 


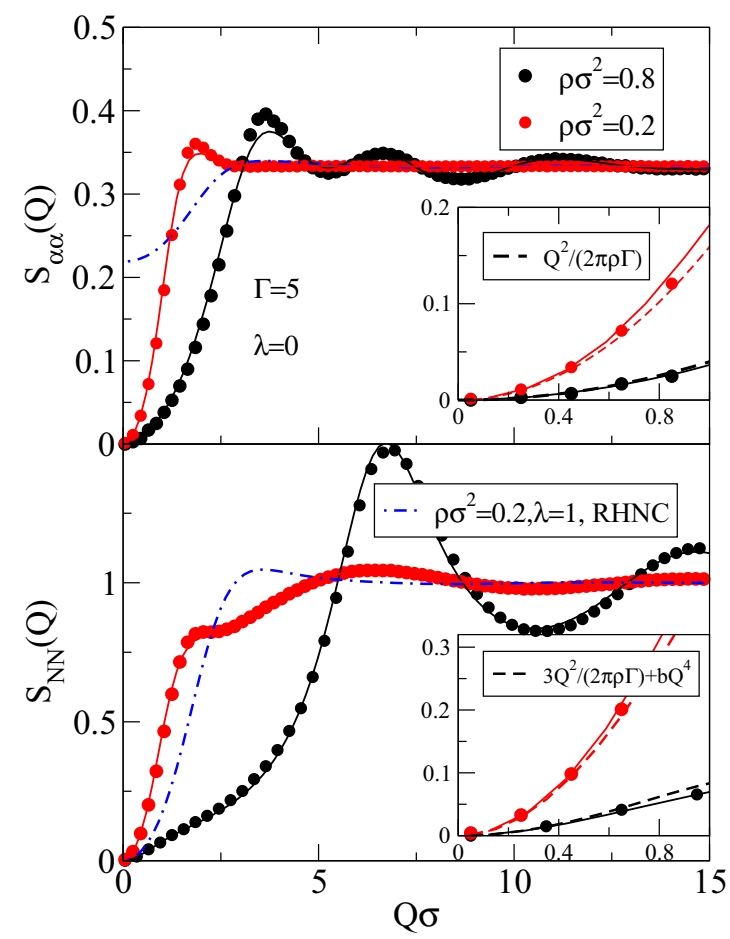

FIG. 2. Total and partial structure factors of our model photoreceptor system displaying multihyperuniformity for low and moderate number densities (see the legend). Solid and dash-dotted curves correspond to theoretical (RHNC) calculations, symbols denote Monte Carlo data. Dashed curves in the insets represent the low- $Q$ regime derived from Eq. (10). Partial structure factors correspond to correlations between like particles. For comparison, we show on blue dash-dotted curves the structure factors for a system displaying only global hyperuniformity $[\lambda=1$ in Eq. (5)].

very long-ranged repulsions between like particles with nonadditive unlike interactions both in the short and long range reproduces the features sought for in our minimal statistical mechanical model of retina.

\section{B. Mimicking avian retina}

How do our model results compare with a real structure factor obtained from a distribution of avian photoreceptors ? One must first bear in mind that in bird retina, five different types of cones [8] are present in unequal numbers, so in principle our model departs significantly from the real situation. Nonetheless, a simple inspection of the experimental structure factors presented in Fig. 9 of Ref. [8] indicates that basically all cone types qualitatively display similar partial structure factors. The global structure factor is qualitatively different, with very little structure at low $Q$ values. Therefore, it seems reasonable to compare our model system with actual experimental results from a qualitative standpoint. To that aim, we have adjusted the coupling constant $\Gamma$ of our effective potential to match the results of [8]. Density is basically coupled to $\Gamma$ (except for subtle hard-core effects not visible in the low $Q$ behavior of the structure factor), so we have set $\rho \sigma^{2}=0.8$ and kept it fixed. As in Ref. [8], $Q$ is scaled with the position of the structure factor maximum, which sets the length scale to the appropriate value in order to ease

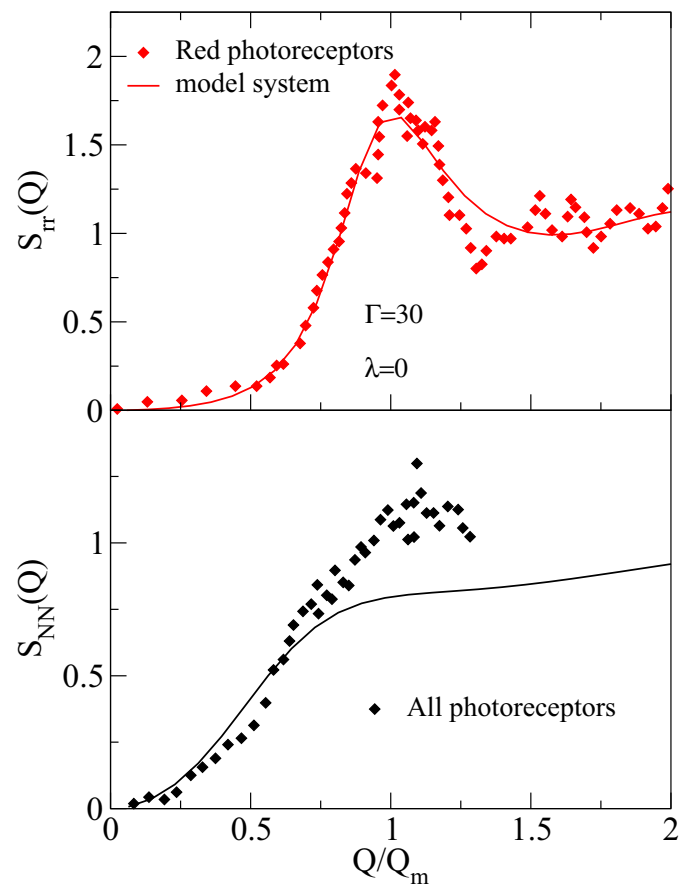

FIG. 3. Total and partial structure factor of the symmetric threecomponent plasma with negative nonadditivity for $\lambda=0, \rho \sigma^{2}=$ $0.8, \Gamma=30$ compared with those from avian photoreceptors from Ref. [8]. In the upper curve, only the red photoreceptors are presented. Solid curves correspond to RHNC calculations, symbols to experimental data [8]. The partial structure factor is normalized to 1 , and the $Q$ axis is scaled with the position of the maximum that is equivalent to adjusting $\sigma$ to the effective experimental value in the photoreceptor correlations.

the comparison. This is equivalent to rescaling the data so as to account for the different size of each cone type. We observe that the behavior of our simple model depicted in Fig. 3 agrees qualitatively with the experimental data. As a matter of fact, even if in Ref. [8] the experimental low- $Q$ behavior seems to follow a linear decay instead of the $Q^{2}$ dependence of our model, the quadratic dependence appears acceptable. As mentioned, the multiscale packing model also proposed by Jiao and co-workers [8] to fit the experimental data displays the same quadratic decay. The other salient feature that is observed in Fig. 3 is the lack of structure of $S_{\mathrm{NN}}(Q)$ for low $Q$ values. This feature is visible both in our simple model (although somewhat enhanced) and in the experimental data. It is apparent that our minimal model is capable of reproducing key features of the spatial patterns displayed by photoreceptors in actual bird retina.

\section{Voronoi analysis}

When thinking of photoreceptors, one must also take into account that their ability to reproduce an image is directly related to the area they sample. This suggests that a Voronoi analysis of our point configurations will provide information as to the sampling area corresponding to each particle. We have therefore performed a characterization of the spatial configurations of our model system using Voronoi tessellations. We have studied the corresponding area distribution 

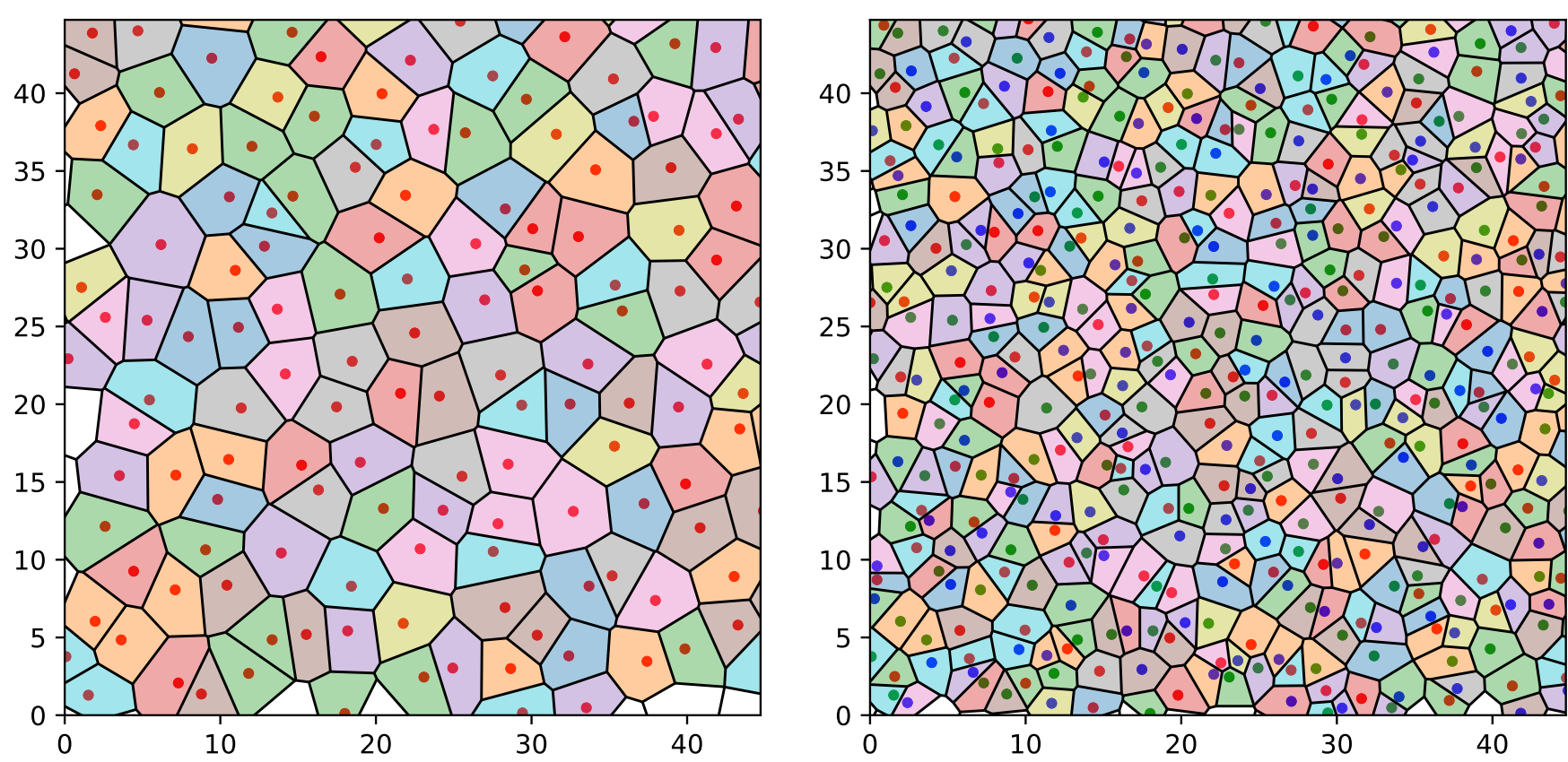

FIG. 4. Voronoi tessellations corresponding to the red cones with $\rho_{\mathrm{red}} \sigma^{2}=0.2 / 3$ (left) of the photoreceptor model and all the photoreceptors (right) for total density $\rho \sigma^{2}=0.2$.

of the Voronoi polygons. To put these results in perspective, we have also performed a corresponding analysis for purely random two-dimensional point configurations, as well as configurations obtained from molecular dynamics simulations for 2D fluids of Lennard-Jones (LJ) particles and LJ particles with added Coulomb repulsion. For these cases, we have used similar density conditions and supercritical temperatures $\left(k_{B} T / \epsilon=2.0\right.$, where $k_{B}$ is Boltzmann's constant and $T$ is the absolute temperature). When referring to $\mathrm{LJ}$ results, $\epsilon$ and $\sigma$ correspond to the well depth and particle size, respectively. In the case of LJ particles with Coulombic repulsion, the competition between short-range attractive and long-range repulsive forces leads to the formation of stable clusters that nonetheless display hyperuniformity. Upon examination of Fig. 4, one can clearly appreciate that in our model system, the Voronoi tessellation exhibits a fairly regular distribution of the polygon areas. A similar observation was made by Legras et al. [38] when analyzing the cone distribution in human retina. However, when comparing with tessellations for LJ fluids or random distributions (left graph in Fig. 5) at similar density, one finds that these have a much larger dispersion in their areas. The effect is even more apparent when competing long-range Coulombic repulsions are added (right graph of Fig. 5). This system, which is also hyperuniform, displays a considerably degree of clustering, and in turn a considerable dispersion in the sizes of the Voronoi polygons.

All these effects can be more quantitatively analyzed by examining the normalized area distributions. These are plotted for our photoreceptor model in Fig. 6 versus the area scaled with the corresponding particle densities, $\rho A$. The corresponding curves for random configurations, LJ fluids, and LJ fluid + Coulombic repulsions are to be found in Fig. 7.

Figure 6 reveals that our model leads to area distributions that are symmetrized with respect to the regular lattice result, $\rho A=1$. It is interesting to note that in all cases the curves apparently follow a Gaussian distribution. This is in sharp contrast with the area distributions for the LJ fluids (with and without Coulombic repulsion) that are plotted in Fig. 7, which are clearly asymmetric. It is important to note that the symmetrization of the area distributions is not a consequence of hyperuniformity. In Ref. [40], it was found that certain stealthy hyperuniform patterns led to asymmetric distributions similar to those of random configurations. This is also illustrated by the shape of the area distribution of Voronoi polygons of LJ particles with added Coulombic repulsions. Although these form hyperuniform patterns, there is a high degree of clustering due to the presence of interparticle attractions. This strongly modifies the local environment leading to asymmetric area distributions. Hyperuniformity is a largescale property and remains unaffected by changes in the shortrange ordering.

The area distribution of Voronoi polygons from random point configurations is known to follow approximately a $\Gamma$ distribution of the form [41]

$$
p d f(x)=\frac{c}{\Gamma(c)} x^{c-1} e^{-c x},
$$

where $c$ is a fitting parameter and $\Gamma(c)$ is the transcendental $\Gamma$-function. In Fig. 7, one can see that the distributions for low-density LJ (with and without Coulombic repulsions) and purely random point configurations can accurately be fitted to Eq. (13). In the upper graph of Fig. 7 we have results for the single species configuration for the LJ fluid at $\rho \sigma^{2}=0.8$. In this instance, correlations are important and no reasonable fit to Eq. (13) is possible. Interestingly, the curves apparently follow a log-normal distribution, a ubiquitous distribution that describes multiple natural growth processes due to accumulation. The corresponding functional form is

$$
p d f(x)=\frac{1}{\sqrt{2 \pi \varsigma^{2} x^{2}}} \exp \left(-\log (x / \mu)^{2} /\left(2 \varsigma^{2}\right)\right),
$$



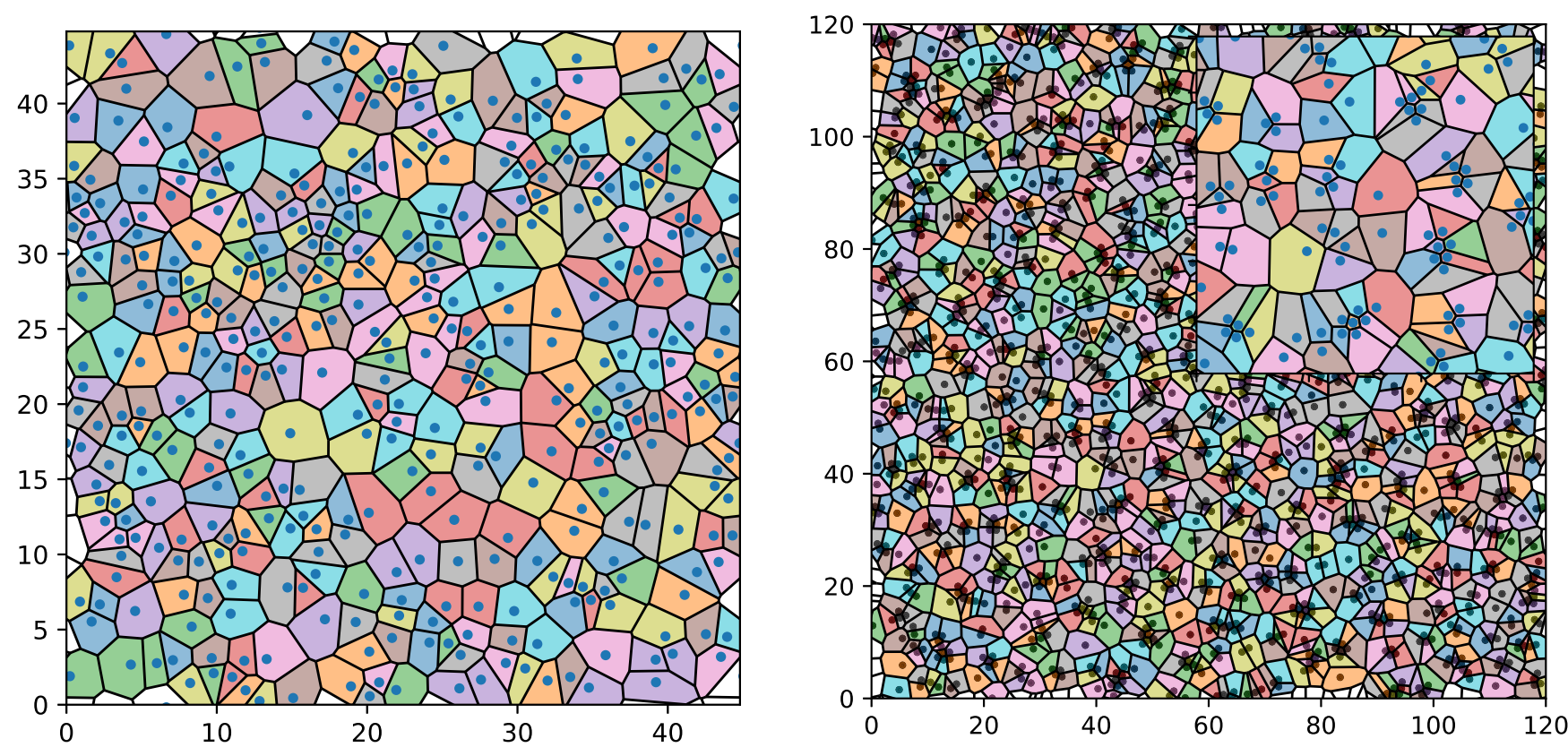

FIG. 5. Voronoi tessellations corresponding to a LJ fluid configuration for $\rho \sigma^{2}=0.2$ (left) and a LJ fluid with added Coulomb repulsion at $\rho \sigma^{2}=0.1111$, and $k_{B} T / \epsilon=2$ (right). The inset on the right figure is a zoom of the central area of the simulation cell. Effects of clustering due to the competing short-range attraction and long-range repulsion are clearly seen. The low-density LJ configuration yields a Voronoi tessellation indistinguishable from that of a random configuration of points.

where $\varsigma$ and $\mu$ are two fitting parameters. Note that the curve is plotted versus $\rho A$ in a log-scale and looks almost Gaussian. This should be exactly the case when the distribution follows Eq. (14). The vertical line represents the $\delta$-function distribution corresponding to the Voronoi tessellation of the regular square lattice, i.e., $\delta(\rho A-1)$, and one can see that the curves are not symmetric with respect to it. For our retina model, the marked symmetry of the Voronoi area distribution is due to the fact that the interactions are monotonic and repulsive, and thus tend to produce very regular local environments. This is illustrated by the uniform linear behavior of number variance $\sigma_{N}^{2}(R)$ for both the global and the single species photoreceptor configurations in our model, as can be seen in Fig. 8. The same linear dependence is found in the experimental photoreceptor distributions (see Fig. 4 in Ref. [8]).

Further clarification is offered by an analysis of the sampling window dependence of the scaled number variance $\sigma_{N}^{2}(R) / R^{2}$, where the $R^{2}$ in the denominator corresponds to the scaling of typical disordered nonhyperuniform systems [19]. We will consider two disordered hyperuniform systems: one composed of LJ particles with added Coulombic repulsion (which exhibits stable clusters), and the other with cut and shifted LJ interactions at $2^{1 / 6} \sigma$, plus Coulombic repulsion (entirely repulsive, no clustering), presented Fig. 9. As a reference, we also plot the values for a plain LJ system at the same density (nonhyperuniform system). The number variance in the latter instance is monotonous and scales quadratically with $R$, whereas for both hyperuniform systems it scales linearly, and in the presence of clustering a marked nonmonotonous behavior is apparent for small sampling windows. This is clear contrast with the monotonic dependence of the results for our photoreceptor model (cf. Fig. 8) and those of real avian cones (Fig. 4 of Ref. [8]).
Finally, we see that hyperuniform configurations that display clustering (and asymmetric area distributions of their Voronoi tessellations) present a number variance with a clear nonmonotonic behavior for small sampling windows. One can then interpret the symmetrization of the area distribution in a disordered media as a consequence of the minimization of repulsive interactions, maximizing the area around each point in the configuration, and reflecting random deviations from the crystalline (ordered hyperuniform) state. This, together with the strong suppression of long-wavelength density and concentration fluctuations, leads to what could possibly be optimal photoreceptor patterns.

\section{CONCLUDING REMARKS}

In summary, we have shown that two key features of the experimental patterns of photoreceptors in bird retina, namely multihyperuniformity and heterocoordination, can be captured by a simple model with logarithmic repulsions between like particles and hard-core exclusions with negative nonadditivity between the unlike ones. The fact that disordered hyperuniform systems represent topological states of matter sharing fluid and crystal-like properties makes them the solution of choice when regular arrangements such as those of arthropod eyes are hampered by the variability of the photoreceptors (e.g., unequal sizes and numbers). Present-day bio-inspired optical devices rely on regular arrangements [5,42]. In certain instances, the combination of different types of receptors and in different numbers might be required, compromising the feasibility of regular arrays of receptors. Disordered multihyperuniformity might then offer an alternative to overcome these difficulties. 


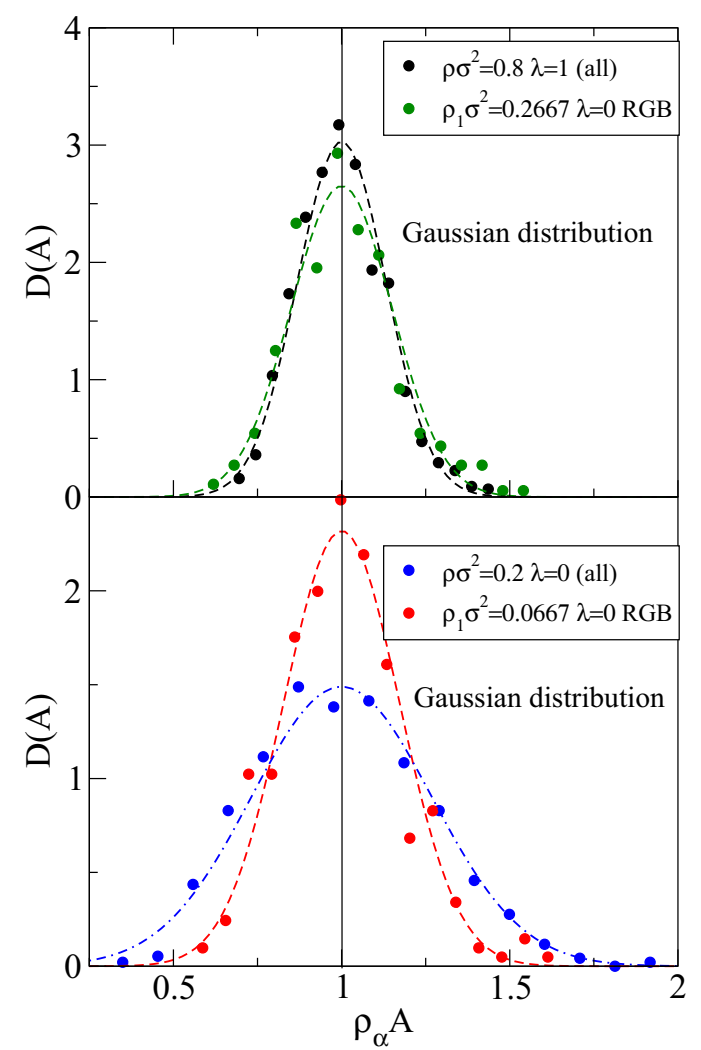

FIG. 6. Scaled area distribution of the Voronoi polygons for hyperuniform states: high-density (upper graph) and low-density (lower graph) single species and global configurations of the threecomponent plasma. Hyperuniformity symmetrizes the area distributions around the value of the square regular lattice $[\delta(\rho A-1)]$, and the curves follow an apparent Gaussian distribution (as shown by the fits represented by dashed curves).

A natural extension of this work should be its application to non-Euclidean geometries. Steps in this direction can be found in the works of Meyra et al. [43] and Božič and Čopar [44] for spherical surfaces. Actually designs on curved surfaces have already been proposed for regular arrays in Ref. [5]. Disordered hyperuniform systems on curved surfaces might well have a potentially larger impact on technological applications. An analysis along these lines of photoreceptor patterns in humans [38] might also be of interest to further our understanding of our complex visual system. In fact, in Ref. [38] it was found that human cones tend to preserve locally hexagonal arrangements, even if the cone patterns are disordered and nonhyperuniform. A preliminary Voronoi analysis of the area distributions taken from Ref. [38] shows that these are also relatively symmetric. However, photoreceptor patterns in human retina (and in birds as well) are also dependent on the eccentricity. This implies that a specific modeling taking into account the positional dependence of photoreceptor composition and density based on experimental information would be needed for a more accurate modeling of each specific retinal region. One should expect to find heterocoordination (probably with the exception of the peripheral region, where there is a marked concentration of rods), otherwise the sampling of light would be degraded. As to

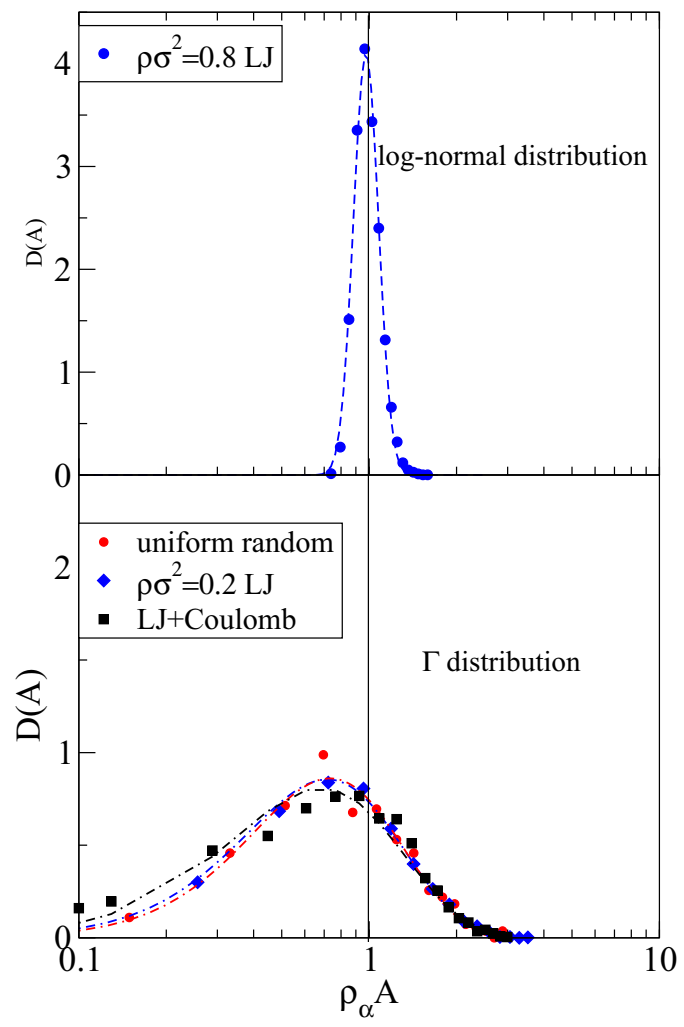

FIG. 7. Scaled area distribution of the Voronoi polygons for LJ + repulsive Coulomb and LJ interacting particles together with random configurations. Dashed lines correspond to fits to Eqs. (13) and (14). The random and low-density LJ configurations (lower graph) agree with $\Gamma$-distributions-Eq. (13)—whereas when correlation effects become appreciable (upper graph) the curves follow an apparent log-normal distribution-Eq. (14). Notice the log scale on the $\rho A$ axis.

multihyperuniformity, this issue deserves further study, given the fact that not all the retinal regions necessarily have the same degree of visual acuity, e.g. the density of cones in the peripheral region of the avian retina is relatively low and inhomogeneous, with a large number of rods taking care of night vision.

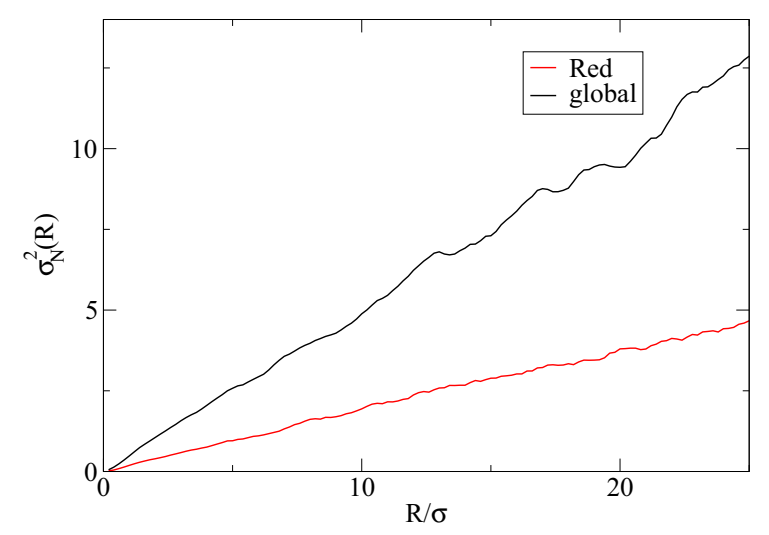

FIG. 8. Number variance $\sigma_{N}^{2}(R)$ dependence of sample window radius, $R$, for both the global and the single species photoreceptor model configurations. 


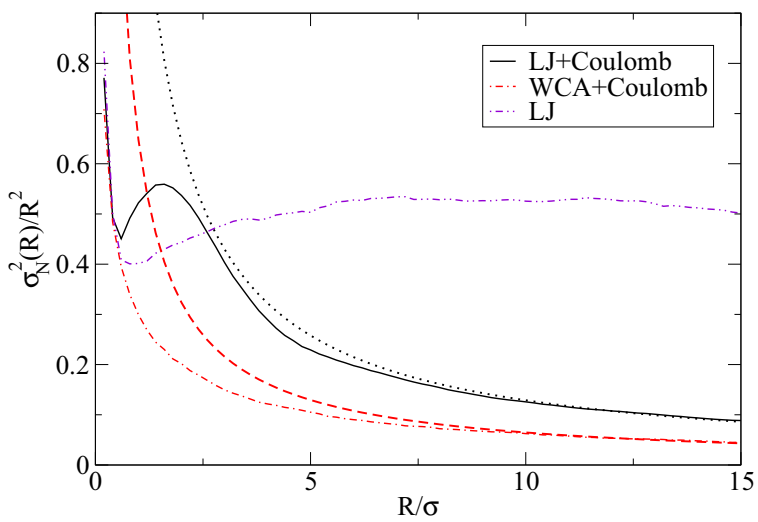

FIG. 9. Scaled number variance $\sigma_{N}^{2}(R) / R^{2}$ dependence of sample window radius, $R$, for $\mathrm{LJ}, \mathrm{LJ}+$ Coulombic repulsion, and purely repulsive soft potential ( $\mathrm{LJ}$ cut and shifted at $r / \sigma=2^{1 / 6}$, i.e., WCA)+Coulombic repulsion. The last two systems are hyperuniform and hence $\sigma_{N}^{2}(R) / R^{2} \rightarrow 0$. Due to the presence of clustering, $\mathrm{LJ}+$ Coulomb repulsion exhibits a clear nonmonotonous behavior for $R / \sigma<4$.

Finally, retinal ganglion cells (RGCs) have a spatial distribution apparently very similar to that of photoreceptors (see, e.g., Fig. 1 in Ref. [45]). An analysis along the lines of the one presented here and in Ref. [8] would be straightforward. One should expect that the ganglion cell distribution would also preserve hyperuniformity, given the fact that RGCs perform the functional integration of the signals from a given number of photoreceptors over a certain spatial scale. The simplest solution to this problem would be to apply a spatial coarsegraining procedure, where the photoreceptors present in a given area are replaced by a single entity, constrained by the condition that the newly generated configuration corresponding to the RGCs retains hyperuniformity. Thus, from the spatial configuration of the model photoreceptor cells in the retina, one could infer the corresponding structure of the inner RGC layer. To that aim, the spatial scale of photoreceptor cells whose signals are to be integrated by a corresponding ganglion cell must be known.

\section{ACKNOWLEDGMENTS}

E.L. acknowledges the support from the Agencia Estatal de Investigación and Fondo Europeo de Desarrollo Regional (FEDER) under Grant No. FIS2017-89361-C3-2-P. E.L. and L.G. were also supported by the European Union's Horizon 2020 Research and Innovation Staff Exchange programme under the Marie Skłodowska-Curie grant agreement No. 734276. S.T. was supported by the National Science Foundation under Award No. DMR-1714722. The authors are grateful to Y. Yiao for kindly providing the structure factor data from Ref. [8].

\section{APPENDIX A: A GENERAL CONDITION FOR MULTIHYPERUNIFORMITY IN MIXTURES}

In the general case of an $n$-component mixture, the Ornstein-Zernike equation in Fourier space reads

$$
\tilde{h}_{i j}(Q)=\tilde{c}_{i j}(Q)+\sum_{l} \tilde{c}_{i l}(Q) \rho_{l} \tilde{h}_{l j}(Q),
$$

where $\tilde{h}_{i j}(Q)$ and $\tilde{c}_{i j}(Q)$ are the Fourier transforms of the total correlation function, $h_{i j}(r)$, and the direct correlation function, $c_{i j}(r)$, respectively, $\rho_{l}$ is the number density of species $l$, and the subscripts denote different species. This gives for the symmetrized partial structure factors

$$
\delta_{i j}+\left(\rho_{i} \rho_{j}\right)^{1 / 2} \tilde{h}_{i j}(Q)=\frac{\mathbf{M}_{i j}(Q)}{|\mathbf{I}-\mathbf{C}|} .
$$

Here $\mathbf{M}_{i j}$ is the $(i j)$ minor of the $\mathbf{I}-\mathbf{C}$ matrix, $\mathbf{I}$ is the identity matrix, $[\mathbf{C}]_{i j}=\left(\rho_{i} \rho_{j}\right)^{1 / 2} \tilde{c}_{i j}(Q)$, and $|\cdots|$ denotes the determinant of the $n \times n$ matrix. When this quantity vanishes as $Q \rightarrow 0$, the system will be multihyperuniform.

Now, if

$$
\lim _{Q \rightarrow 0} \tilde{c}_{i i}(Q) \propto Q^{-\alpha} \forall i
$$

with $\alpha>0$, then

$$
\lim _{Q \rightarrow 0} M_{i j}(Q) \propto Q^{-(n-1) \alpha} .
$$

Additionally, one might expand the denominator in (A2) [46],

$$
|\mathbf{I}-\mathbf{C}|=1-|\mathbf{C}|-\sum_{i=1}^{n-1} \Gamma_{n}^{i}\left|\mathbf{C} / \mathbf{I}^{i}\right|,
$$

where $\Gamma_{n}^{i}\left|\mathbf{C} / \mathbf{I}^{i}\right|$ is the sum of the combination of determinants in which the $i$ th row of $\mathbf{C}$ is replaced by the corresponding row of $\mathbf{I}$. Now, when $|\mathbf{C}| \neq 0$, it turns out that

$$
\lim _{Q \rightarrow 0}|\mathbf{I}-\mathbf{C}|=-\lim _{Q \rightarrow 0}|\mathbf{C}| \propto Q^{-n \alpha}
$$

It can be shown that

$$
|\mathbf{C}|=\left(\prod_{i=1}^{m} \rho_{i}\right)|\mathbf{c}|
$$

with $[\mathbf{c}]_{i j}=\tilde{c}_{i j}(Q)$. When Eq. (A3) is satisfied, then $\quad \lim _{Q \rightarrow 0} \tilde{c}_{i j}(Q)=-\lim _{Q \rightarrow 0} \beta \tilde{u}_{i j}(Q)$, by which $\lim _{Q \rightarrow} \beta \tilde{u}_{i j}(Q) \propto Q^{-\alpha}$. Using Eqs. (A4), (A6), and (A2), one gets

$$
\lim _{Q \rightarrow 0}\left(\delta_{i j}+\left(\rho_{i} \rho_{j}\right)^{1 / 2} \tilde{h}_{i j}(Q)\right) \propto Q^{\alpha} \forall(i, j),
$$

and therefore all the structure factors will vanish as $Q \rightarrow 0$. This implies that the system will be multihyperuniform if the conditions

$$
\begin{gathered}
\lim _{Q \rightarrow 0} \beta \tilde{u}_{i i}(Q) \propto Q^{-\alpha}, \\
\lim _{Q \rightarrow 0}|\tilde{\mathbf{u}}(Q)| \neq 0,
\end{gathered}
$$

are satisfied, where $[\mathbf{u}]_{i j}=\tilde{u}_{i j}(Q)$. This is a generalization of Eq. (7) in Ref. [35].

\section{APPENDIX B: ASYMPTOTICS IN THE SYMMETRIC THREE-COMPONENT CASE}

In the case of mixtures, one must monitor the small wavenumber behavior of the partial structure factors. These quantities can be expressed in terms of the corresponding atom-atom total correlation functions as

$$
S_{i j}(Q)=x_{i}\left(\delta_{i j}+\rho x_{j} \tilde{h}_{i j}(Q)\right) .
$$


Now, when all densities are identical, such that $\rho_{i}=\rho / 3 \forall i$, where $\rho$ is the total number density, and the interactions are given by Eq. (5), one can solve Eq. (A1) in Fourier space and obtain for the partial structure factors

$$
\begin{aligned}
& \lim _{Q \rightarrow 0} S_{i i}(Q)=Q^{2} \frac{Q^{2}-\rho_{1}\left(\tilde{c}_{11}^{R}+\tilde{c}_{12}^{R}\right) Q^{2}+2 \pi \rho_{1} \Gamma(1+\lambda)}{Q^{4} \operatorname{det}|\mathbf{I}-\mathbf{C}|}, \\
& \lim _{Q \rightarrow 0} S_{i j}(Q)=x_{i}\left(\delta_{i j}+\rho x_{j} Q^{2} \frac{\tilde{c}_{i j}^{R} Q^{2}-2 \pi \rho_{i} \Gamma \lambda}{Q^{4} \operatorname{det}|\mathbf{I}-\mathbf{C}|}\right),
\end{aligned}
$$

with $i \neq j$, and with the determinant in the denominator given by

$$
\begin{aligned}
Q^{4} \operatorname{det}|\mathbf{I}-\mathbf{C}|= & {\left[Q^{2}-\rho_{i}\left(\tilde{c}_{i i}^{R}-\tilde{c}_{i j}^{R}\right) Q^{2}+2 \pi \rho_{1} \Gamma(1-\lambda)\right] } \\
& \times\left[Q^{2}-\rho_{1}\left(\tilde{c}_{i i}^{R}+2 \tilde{c}_{i j}^{R}\right) Q^{2}+2 \pi \rho_{i} \Gamma(1+2 \lambda)\right],
\end{aligned}
$$

where the Fourier transforms of the direct correlation functions are separated into a short-range component $\left[\tilde{c}_{i i}^{R}(Q)\right.$ and $\left.\tilde{c}_{12}^{R}(Q)\right]$ and Coulomb-like contributions, namely $\tilde{c}_{i i}(Q)=\tilde{c}_{i i}^{R}(Q)+2 \pi \Gamma / Q^{2}, \forall i$, and $\tilde{c}_{i j}=\tilde{c}_{i j}^{R}(Q)+$ $2 \pi \lambda \Gamma / Q^{2}, \forall i \neq j$. We know that the zero-wave-number limit of the short-range components is always finite and nonzero.

From Eq. (B2) we find that whenever $\lambda \neq 1$ and $\lambda \neq-1 / 2$, then $\lim _{Q \rightarrow 0} S_{i i}(Q) \propto Q^{2}$ for all species, that is, the system will be multihyperuniform. Also, in the fully symmetric case, $\lambda=-1 / 2$ corresponds precisely to a two-dimensional Coulomb electrolyte, where we have $1 / 3$ of the particles with charge +1 and $2 / 3$ of the particles with charge $-1 / 2$. This system will then behave as a "regular fluid" and does not even display global hyperuniformity due to the screening effects [35]. The $\lambda=1$ case will not be multihyperuniform, but it will preserve the global hyperuniformity of the single-component two-dimensional Coulomb plasma.
[1] P. Hegemann, Planta 203, 265 (1997).

[2] D. F. Ready, T. E. Hanson, and S. Benzer, Dev. Biol. 53, 217 (1976).

[3] D. K. Lubensky, M. W. Pennington, B. I. Shraiman, and N. E. Baker, Proc. Natl. Acad. Sci. USA 108, 11145 (2011).

[4] D. P. Petersen and D. Middleton, Inf. Control 5, 279 (1962).

[5] Y. M. Song, Y. Xie, V. Malyarchuk, J. Xiao, I. Jung, K.-J. Choi, Z. Liu, H. Park, C. Lu, R.-H. Kim, R. Li, K. B. Crozier, Y. Huang, and J. A. Rogers, Nature (London) 497, 95 (2013).

[6] Y. Zheng, L. Song, J. Huang, H. Zhang, and F. Fang, Opt. Lett. 44, 4143 (2019).

[7] P. A. Raymond and L. K. Barthel, Int. J. Dev. Biol. 48, 935 (2004).

[8] Y. Jiao, T. Lau, H. Hatzikirou, M. Meyer-Hermann, J. C. Corbo, and S. Torquato, Phys. Rev. E 89, 022721 (2014).

[9] R. F. Dunn, J. Ultrastruct. Res. 16, 672 (1966).

[10] These five cone types include one double cone type that is assumed to mediate achromatic motion perception [13], and four single cone types building a tetrachromatic color sensing device, covering wavelengths from red to ultraviolet [14].

[11] M. Ruggeri, J. C. Major, C. McKeown, R. W. Knighton, C. A. Puliafito, and S. Jiao, Invest. Opthalmol. Vis. Sci. 51, 5789 (2010).

[12] M. B. Toomey and J. C. Corbo, Front. Neural Circuit. 11, 97 (2017).

[13] Y. A. Kram, S. Mantey, and J. C. Corbo, PLoS ONE 5, e8992 (2010).

[14] J. Withgott, BioScience 50, 854 (2000).

[15] M. Seifert, T. Baden, and D. Osorio, Seminars Cell Develop. Biol. (2020), doi:10.1016/j.semcdb.2020.03.011.

[16] T. H. Goldsmith, J. S. Collins, and S. Licht, Vision Res. 24, 1661 (1984).

[17] B. A. Moore, P. Baumhardt, M. Doppler, J. Randolet, B. F. Blackwell, T. L. DeVault, E. R. Loew, and E. Fernandez-Juricic, J. Exp. Biol. 215, 3442 (2012).

[18] D. Frenkel, Introduction to colloidal systems, in Soft Condensed Matter Physics in Molecular and Cell Biology, edited by W. C.
K. Ponn and D. Andelman (Taylor \& Francis, Boca Raton, FL, 2006), pp. 19-47.

[19] S. Torquato and F. H. Stillinger, Phys. Rev. E 68, 041113 (2003).

[20] S. Torquato, J. Phys. : Condens. Matter 28, 414012 (2016); Phys. Rep. 745, 1 (2018).

[21] M. Florescu, S. Torquato, and P. J. Steinhardta, Proc. Natl. Acad. Sci. USA 106, 20658 (2009).

[22] L. S. Froufe-Pérez, M. Engel, J. J. Sáenz, and F. Scheffold, Proc. Natl. Acad. Sci. USA 114, 9570 (2017).

[23] O. Leseur, R. Pierrat, and R. Carminati, Optica 3, 763 (2016).

[24] Q. Le Thien, D. McDermott, C. J. O. Reichhardt, and C. Reichhardt, Phys. Rev. B 96, 094516 (2017).

[25] G. Zhang, F. H. Stillinger, and S. Torquato, J. Chem. Phys 145, 244109 (2016).

[26] D. Chen and S. Torquato, Acta Mater. 142, 152 (2018).

[27] Y. Xu, S. Chen, P.-E. Chen, W. Xu, and Y. Jiao, Phys. Rev. E 96, 043301 (2017).

[28] B.-Y. Wu, X.-Q. Sheng, and Y. Hao, PloS One 12, e0185921 (2017).

[29] R. Dickman and S. D. da Cunha, Phys. Rev. E 92, 020104(R) (2015).

[30] R. Garcia-Millan, G. Pruessner, L. Pickering, and K. Christensen, Europhys. Lett. 122, 50003 (2018).

[31] D. Hexner and D. Levine, Phys. Rev. Lett. 114, 110602 (2015).

[32] J. H. Weijs, R. Jeanneret, R. Dreyfus, and D. Bartolo, Phys. Rev. Lett. 115, 108301 (2015).

[33] E. Tjhung and L. Berthier, Phys. Rev. Lett. 114, 148301 (2015).

[34] A. Mayer, V. Balasubramanian, T. Mora, and A. M. Walczak, Proc. Natl. Acad. Sci. USA 112, 5950 (2015).

[35] E. Lomba, J.-J. Weis, and S. Torquato, Phys. Rev. E 97, 010102(R) (2018).

[36] E. Lomba, J. J. Weis, and S. Torquato, Phys. Rev. E 96, 062126 (2017).

[37] F. Lado, Phys. Rev. B 17, 2827 (1978). 
[38] R. Legras, A. Gaudric, and K. Woog, PloS One 13, e0191141 (2018).

[39] S. Leeuw and J. W. Perram, Physica A 113, 546 (1982).

[40] G. Zhang, F. H. Stillinger, and S. Torquato, Phys. Rev. E 92, 022119 (2015).

[41] M. Ferraro and L. Zaninetti, Physica A 391, 4575 (2012).

[42] M. Garcia, C. Edmiston, T. York, R. Marinov, S. Mondal, N. Zhu, G. P. Sudlow, W. J. Akers, J. Margenthaler, S. Achilefu,
R. Liang, M. A. Zayed, M. Y. Pepino, and V. Gruev, Optica 5, 413 (2018).

[43] A. G. Meyra, G. J. Zarragoicoechea, A. L. Maltz, E. Lomba, and S. Torquato, Phys. Rev. E 100, 022107 (2019).

[44] A. L. Božič and S. Čopar, Phys. Rev. E 99, 032601 (2019).

[45] T. Dolan and E. Fernández-Juricic, Brain, Behav. Evol. 75, 111 (2010).

[46] S. J. Xu, M. Darouach, and J. Schaefers, IEEE Trans. Automat. Contr. 38, 1671 (1993). 